\title{
Innovative Design and Analysis of an Electrically Small Reconfigurable Antenna for GPS and Blue Tooth Applications
}

\author{
Harshitha Reddy Katireddy \\ Department of ECE \\ Koneru Lakshmaiah Education Foundation \\ Vaddeswaram, Guntur, Andhra Pradesh, India \\ govardhanee_ec@kluniversity.in
}

\author{
M. Venkata Narayana \\ Department of ECE \\ Koneru Lakshmaiah Education Foundation \\ Vaddeswaram, Guntur, Andhra Pradesh, India \\ mvn@kluniversity.in
}

\author{
Govardhani Immadi \\ Department of ECE \\ Koneru Lakshmaiah Education Foundation \\ Vaddeswaram, Guntur, Andhra Pradesh, India \\ govardhanee_ec@kluniversity.in
}

\begin{abstract}
In this paper, an electrically small, planar antenna with broad side radiation pattern is presented. The design contains a dipole and a segmented circular loop which works equivalent to that of a magnetic dipole. A circular patch with slots is used to provide impedance matching. In general, electrically small antennas suffer from narrow bandwidth. In this paper, the reconfigurability of the small antenna for two different applications, $1.5 \mathrm{GHz}$ and $2.4 \mathrm{GHz}$, is discussed. This reconfigurability was achieved by using a BAR 64-03W pin diode to adjust the resonant frequency. Two reconfigurable frequency bands were achieved at $1.5 \mathrm{GHz}$ and $2.4 \mathrm{GHz}$ with broad side radiation patterns.
\end{abstract}

Keywords-electrically small antenna; PIN diode; end fire radiation pattern; NFRP elements

\section{INTRODUCTION}

The components used in wireless communication systems such as antennas, filters, multiplexers, modulators, etc. are continuously miniaturized and intelligentized [1-3]. Frequency reconfigurable electrically small antennas are often suitable and are used for compact, wearable devices $[4,5]$. An antenna with a broad front-to-back ratio and an equivalent pattern of $\mathrm{E}$ and $\mathrm{H}$ plane radiation is shown in [6]. The research groups in [7-10] and [11-15] have extensively researched antennas with the combination of electric and magnetic dipoles. The quarter wavelength patch with vertical orientation is commonly used in [7] as a magnetic dipole, which is coupled with the electric dipole to design complementary antennas with a wavelength of $0.25 \lambda_{0}$ [8-12]. NFRP elements were used in [13] to design electric and magnetic dipoles, contributing to the design of dual band electrically small antennas. Most of the tiny electrically small antennas are not reconfigurable except from [4] and [5]. Three magnetic NFRP elements were paired with two electric NFRP elements in [5] to achieve the reconfigurability of their radiation pattern. A planar, reconfigurable complementary antenna with a single layer pattern was indicated in [16]. In addition to the reconfigurability of polarization and radiation reconfigurability patterns, due to its powerful spectrum use, frequency reconfigurability has gained a lot of attention [1720]. Utilizing appropriate antenna size, different frequency reconfigurable monopole antennas [17-19], patch antennas [2022] and quasi-Yagi Uda antennas [23-24] have been created. In order to obtain a unidirectional radiation pattern which increases the antenna size, a broad ground plane is required. By eliminating the ground plane, the complementary Huygens source antenna is used to achieve high front-to-back ratios with less distance. This paper introduces an electrically small reconfigurable antenna. To produce the magnetic dipole that is combined with the rectangular bar electric dipole to obtain the end fire radiation pattern, a segmented circular loop is used. This antenna is $35 \mathrm{~mm} \times 35 \mathrm{~mm} \times 1.6 \mathrm{~mm}$ in size and has a single coating. In addition, the design is expanded by inserting a pin diode in the electric dipole to achieve frequency reconfigurability. Two resonant bands were obtained at 1.5 and $2.4 \mathrm{GHz}$ and the end fire radiation pattern was maintained. This antenna was fabricated in order to obtain a practical realization of the results. A good agreement between the simulated and the experimental results was obtained.

\section{ANTENNA MECHANISM}

The magnetic dipole is considered to be a loop with a uniform current distribution. Due to its low radiation resistance and high reactance, its impedance matching is poor. The diameter of the outer loop is $16.8 \mathrm{~mm}$ at the resonant frequency and the current distribution is uniform and flows along the $\mathrm{Y}$-axis (Figure 1). The electrical and magnetic dipoles are excited in a perpendicular manner in order to design this planar, electrically small antenna. The strip line and the 
circular loop on the top and bottom layers of the substrate are mounted at this end. The length and width of the patch are calculated by (1)-(3).

$$
W=\frac{V_{O}}{2 F_{r}} \sqrt{\frac{2}{\varepsilon_{\mathrm{r}}+1}}
$$

where $\mathrm{v}_{0}$ is the velocity of light in free space.

$$
\begin{gathered}
L=\frac{C}{2 F_{r} \sqrt{\varepsilon_{r e f f}}}-2 \Delta l \\
\Delta l=0.412 h \frac{\left(\varepsilon_{r e f f}+0.03\right)(w+0.26 h)}{\left(\varepsilon_{r e f f}-0.258\right)(w+0.8 h)}
\end{gathered}
$$

where $\Delta l$ is the extension in length due to fringing effects.

The effective dielectric constant is given by:

$$
\varepsilon_{\text {reff }}=\frac{\varepsilon_{r}+1}{2}+\frac{\varepsilon_{r}-1}{2}\left[1+\left[\frac{12 h}{w}\right]\right]^{-1 / 2}
$$

The actual length and width of the ground plane can be calculated by (5) and (6):

$$
\begin{gathered}
L_{g}=6 h+L \\
W_{g}=6 h+W
\end{gathered}
$$

where $L$ is the length of the patch, $W$ its width, and $h$ is the thickness of the substrate.

Length and width of the feed line are calculated by:

$$
B=\frac{60 \Pi^{2}}{\sqrt{\varepsilon_{r} Z_{C}}}
$$

where $Z_{c}$ is the characteristic impedance and $\varepsilon_{r}$ the relative permittivity of the substrate.

$$
L_{f}=\frac{\lambda_{0}}{4 \sqrt{\varepsilon_{e f f}}}
$$

The radius of the patch is calculated by:

$$
a=\frac{F}{\sqrt{1}+\frac{2 h}{\Pi \varepsilon_{r}}\left[\ln \left(\frac{\Pi f}{2 h}\right)+1.7726\right]}
$$

Small antennas are those in which the largest dimension is less than the one-tenth of the wave length $(\lambda / 10)$. Small antennas are those having $K . a<1$, where $K=$ wave number $=\frac{2 \pi}{\lambda}=0.0314$. For the proposed antenna the obtained $K . a$ value is: $K . a=0.0314 \times 16.8=0.527<1$.

\section{ANTENNA DESIGN CONSIDERATION}

Figure 1 represents the configuration of the electrically small planar antenna. It mainly consists of a segmented loop and strip line fabricated on a FR4 substrate with 4.4 relative permittivity, $1.6 \mathrm{~mm}$ thickness, $35 \mathrm{~mm}$ length, and $35 \mathrm{~mm}$ width. The top layer of the antenna is fabricated with a strip line and it is fed by an edge feed acting as an electric dipole. The bottom layer of the antenna, i.e. in the ground plane, contains an outer circle with radius $R_{4}$ and an inner circle with radius $R_{3}$ (see Table I). The proposed antenna was designed and analyzed experimentally. The prototype of the antenna was fabricated and the result was verified by a vector network analyzer (Figure 2). Figure 3 shows the fabricated antenna.

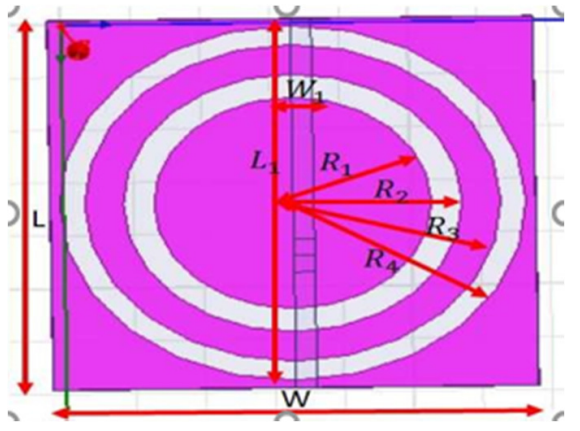

Fig. 1. The designed planar electrically small antenna.

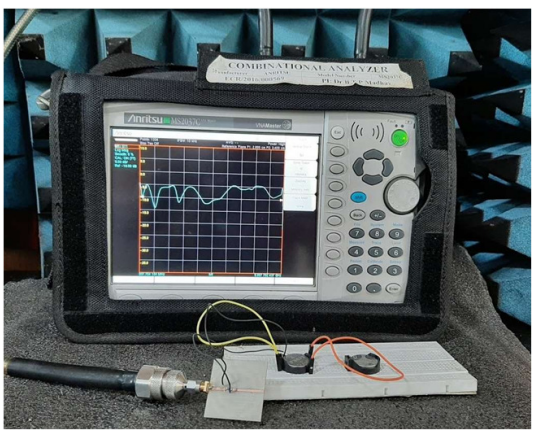

Fig. 2. Antenna parameter testing using VNA

TABLE I. DIMENSIONS OF THE DESIGNED PLANAR ELECTRICALLY SMALL ANTENNA

\begin{tabular}{|c|c|c|c|c|c|c|c|c|}
\hline Param eter & $L$ & $W$ & $L_{1}$ & $W_{1}$ & $R_{1}$ & $R_{2}$ & $R_{3}$ & $R_{4}$ \\
\hline Value (mm) & 35 & 35 & 35 & 1.5 & 10 & 12.2 & 15 & 16.8 \\
\hline
\end{tabular}

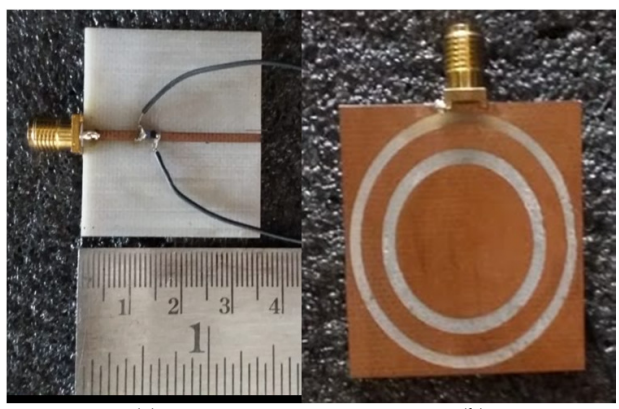

(a)

(b)

Fig. 3. Images showing the prototype of the proposed planar electrically small antenna: (a) top view, (b) bottom view.

\section{RESULTS AND DISCUSSION}

Return loss is the most preferred parameter to test an antenna. Instead of using 2 different antennas at different frequencies, a single antenna can switch between 2 frequencies. In the proposed model, the antenna can switch between $1.5 \mathrm{GHz}$ and $2.4 \mathrm{GHz}$. Figure 4 shows the return loss of the antenna when the diode is in the OFF state. During the OFF state, the antenna resonates at $2.4 \mathrm{GHz}$, with a return loss value of $-15.8 \mathrm{dbm}$ for the simulated and $-13.01 \mathrm{dbm}$ for the actual antenna. Figure 5 represents the equivalent circuit diagram of a pin diode in ON and OFF state. During the ON state, the antenna resonates at $1.5 \mathrm{GHz}$ and only the resistor and inductor values are used. During the OFF state, the antenna resonates at 
$2.4 \mathrm{GHz}$ and the resistor, capacitor, and inductor values are used. Figure 6 shows the return loss of the antenna when the diode is at the $\mathrm{ON}$ state. The proposed antenna resonates at $1.5 \mathrm{GHz}$ with a return loss value of $-15.07 \mathrm{~dB}$ for the simulated model and $-13.08 \mathrm{~dB}$ for the fabricated model.

Voltage Standing Wave Ratio (VSWR) is a significant parameter for estimating the behavior of the RF components at high frequencies. The proposed antenna resonates at $2.4 \mathrm{GHz}$ with circular polarization when the diode is in the OFF state. Figure 8 shows the 3D radiation pattern of the antenna with broad side radiation. Maximum radiation exists in the $X Y$ plane. The maximum possible gain is about $1 \mathrm{~dB}$ at $\theta=0^{\circ}$. Figures 9-10 represent the patterns of the antenna at various azimuthal and elevation planes.

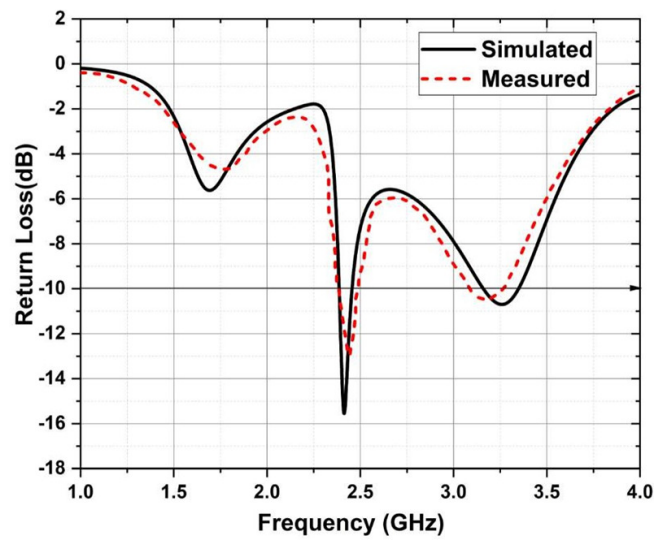

Fig. 4. Return loss of the antenna when the diode is in the OFF state.

(a)

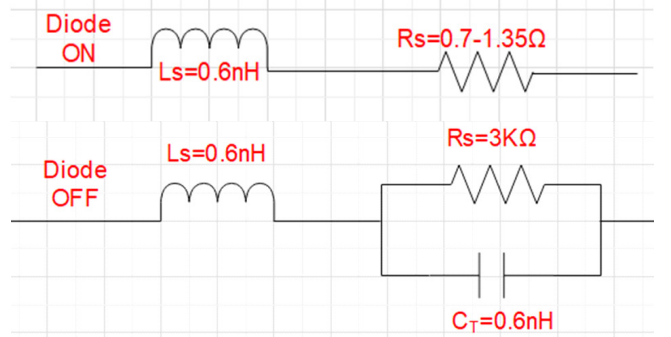

Fig. 5. Equivalent circuit diagram of a pin diode. (a) ON, (b) OFF.

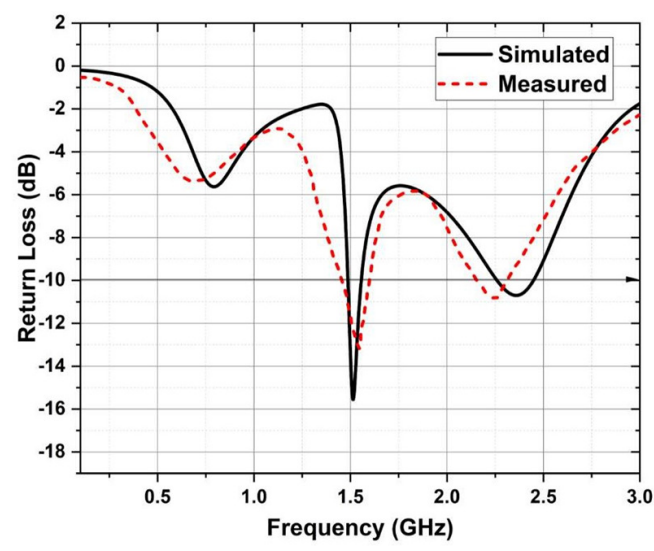

Fig. 6. Return loss when the diode is in the ON state.

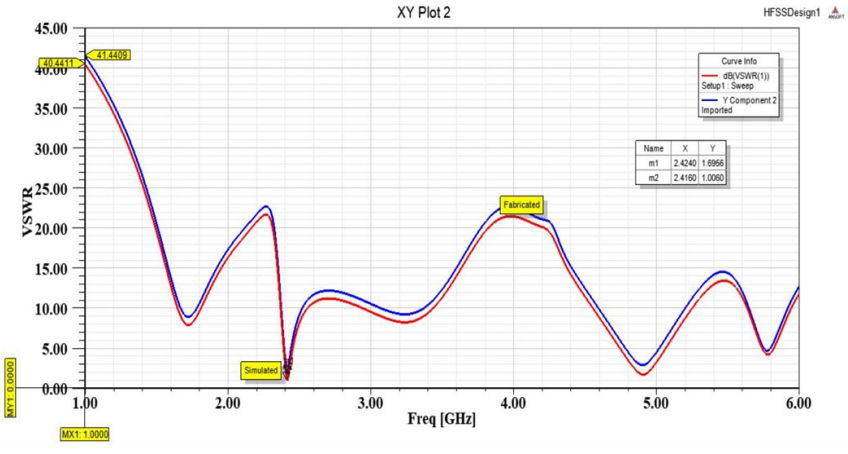

Fig. 7. VSWR of the antenna.

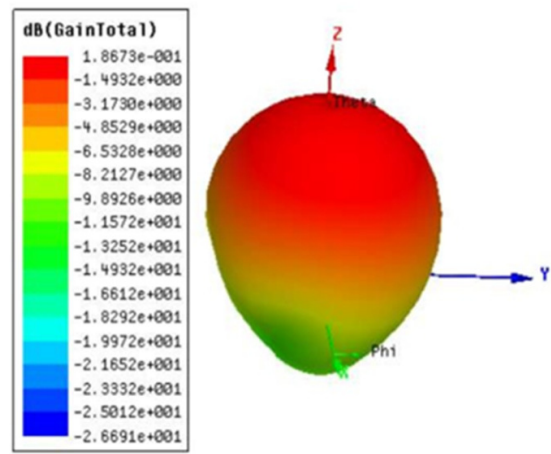

Fig. 8. 3D radiation pattern of the antenna

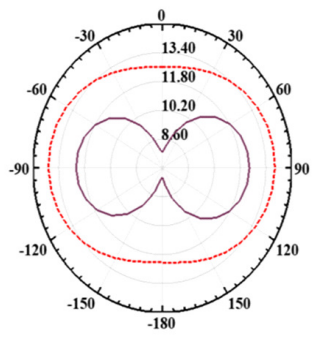

(a)

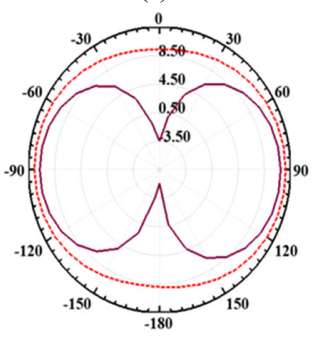

(c)

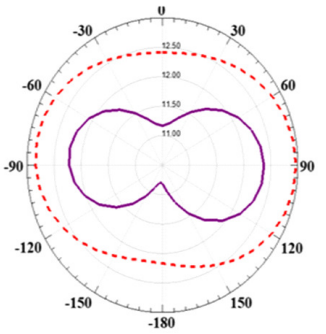

(e)

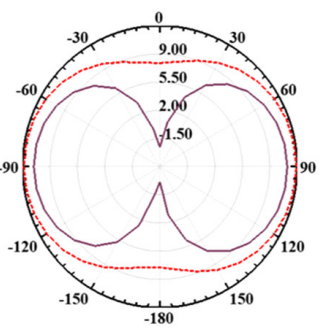

(b)

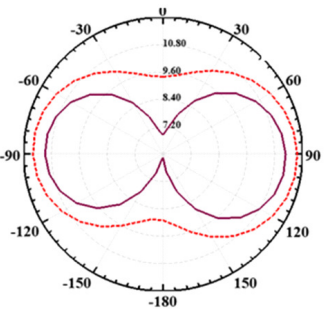

(d)

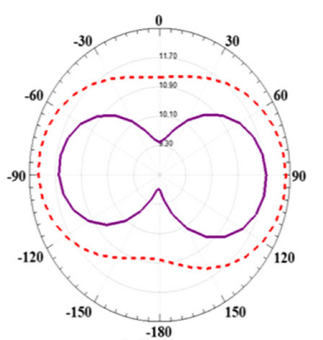

(f)
Fig. 9. Azimuth pattern at $\theta=$ (a) $0^{\circ}$, (b) $30^{\circ}$, (c) $60^{\circ}$, (d) $90^{\circ}$, (e) $120^{\circ}$, (f) $180^{\circ}$. Purple line: simulated results, red line: measured results. 


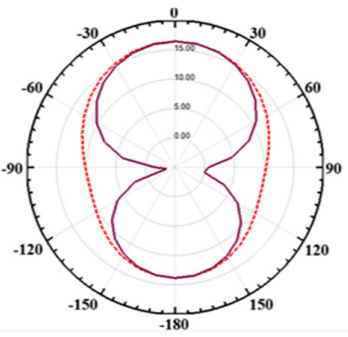

(a)

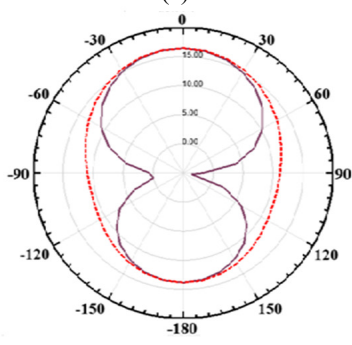

(c)

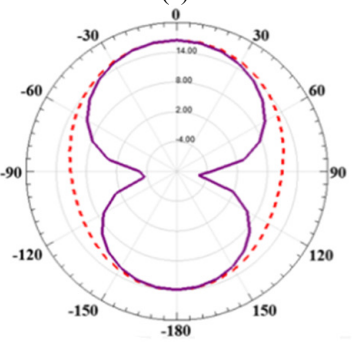

(e)

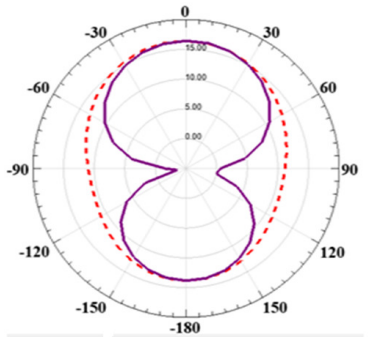

(b)

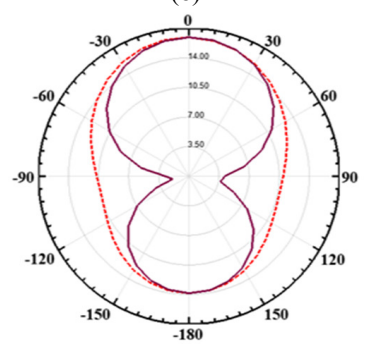

(d)

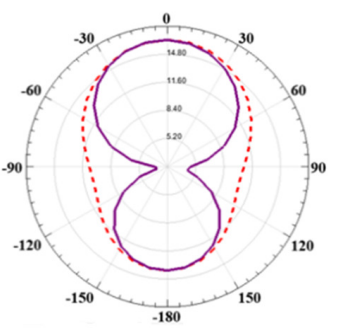

(f)
Fig. 10. Elevation pattern at $\varphi=$ (a) $0^{\circ}$, (b) $30^{\circ}$, (c) $60^{\circ}$, (d) $90^{\circ}$, (e) $120^{\circ}$, (f) $180^{\circ}$. Purple line: simulated results, red line: measured results.

The comparison with other proposed designs in Table II shows that that the proposed antenna offers better results in terms of return loss and gain.

TABLE II. PERFORMANCE COMPARISON OF THE PROPOSED ANTENNA WITH OTHER, KNOWN ANTENNAS

\begin{tabular}{|c|c|c|c|c|c|}
\hline Ref & Substrate & Reconfigurable & $\begin{array}{c}\text { Area } \\
\left.\mathbf{( m m}^{3}\right)\end{array}$ & $\begin{array}{c}\text { Center } \\
\text { Frequency }\end{array}$ & $\begin{array}{c}\text { Gain } \\
\mathbf{( d b})\end{array}$ \\
\hline$[1]$ & $\begin{array}{c}\text { FR-4 } \\
\text { Epoxy }\end{array}$ & Frequency & $45 \times 40 \times 1.6$ & $\begin{array}{c}1.28 \mathrm{GHz} \\
1.62 \mathrm{GHz}\end{array}$ & ------- \\
\hline$[6]$ & $\begin{array}{c}\text { RT } \\
\text { Duroid } \\
5880\end{array}$ & Frequency & $45 \times 38 \times 0.254$ & $2.4 \mathrm{GHz}$ & ------ \\
\hline$[7]$ & Polyimide & Frequency & $45 \times 35 \times 3.2$ & $1.6 \mathrm{GHz}$ & $0.9 \mathrm{~dB}$ \\
\hline$[8]$ & FR4 & Frequency & $95 \times 100 \times 14$ & $1.71 \mathrm{GHz}$ & ------- \\
\hline Proposed & FR4 & Frequency & $35 \times 35 \times 1.6$ & $\begin{array}{l}1.5 \mathrm{GHz} \\
2.4 \mathrm{GHz}\end{array}$ & $1.86 \mathrm{~dB}$ \\
\hline
\end{tabular}

\section{CONCLUSION}

Based on the complementary concept, an electrically small, coplanar antenna was realized by combing the equivalent magnetic dipole of a segmented loop and the electric dipole of a strip line. The antenna is designed on a FR4 epoxy substrate, having a small area of $35 \times 35 \times 1.6 \mathrm{~mm}^{3}$. The antenna showed satisfying broadside radiation performance, exhibiting a gain of $1.86 \mathrm{~dB}$. It has been found that the resonance frequencies of both the electric dipole and the magnetic dipole (segmented loop) can be easily tuned with PIN diodes. Therefore, a frequency-reconfigurable complementary antenna was further developed. Two reconfigurable broadside radiation bands have been achieved at 1.5 and $2.4 \mathrm{GHz}$.

\section{REFERENCES}

[1] W. B. Hong, K.-H. Back, and S. Ko, "Millimeter- wave 5G antennas for smartphones: overview and experimental demonstration," IEEE Transactions on Antennas and Propagation, vol. 65, no. 12, pp. 62506261, December 2017, https://doi.org/10.110 9/TAP.2017.2740963.

[2] H. Maema and T. Fukusako, "Radiation efficiency improvement for electrically small and low-profile antenna by stacked elements," IEEE Antennas and Wireless Propagation Letters, vol. 13, pp. 305-308, January 2014, https://doi.org/10.1109/LAWP.2014.2305304.

[3] M.-C. Tang, B. Zhou, and R. W. Ziolkowski, "Low- profile, electrically small, Huygens source antenna with pattern-reconfigurability that covers the entire azimuthal plane," IEEE Transactions on Antennas and Propagation, vol.65, no. 3, pp. 1063-1072, March 2017, https://doi.org/10.1109/TAP.2016.2647712.

[4] A. Chlavin, "A new antenna feed having equal E -and H-plane patterns," Transactions of the IRE Professional Group on Antennas and Propagation, vol. 2, no. 3, pp. 113-119, Jul. 1954, https://doi.org/ 10.1109/T-AP.1954.27983.

[5] L. Siu, H. Wong, and K. M. Luk, "A dual-polarized magneto-electric dipole antenna with dielectric loading," IEEE Transactions on Antennas and Propagation, vol. 57, no. 3, pp. 616-623, March 2009, https://doi.org/10.1109/TAP.2009.2013430.

[6] H. Wong, K. M. Mak, and K. M. Luk, "Wideband shorted bowtie patch antenna with electric dipole," IEEE Transactions on Antennas and Propagation, vol. 56, no.7, pp. 2098-2101, July 2008, https://doi.org/ 10.1109/TAP.2008.924765.

[7] L. Ge and K. M. Luk, "A low-profile magneto- electric dipole antenna," IEEE Transactions on Antennas and Propagation, vol. 60, no. 4, pp. 1684-1689, January 2012, https://doi.org/10.1109/LAWP.2016. 2519942.

[8] L. Ge and K. M. Luk, "Linearly polarized and dual- polarized magnetoelectric dipole antennas with reconfigurable beamwidth in the H-plane," IEEE Transactions on Antennas and Propagation, vol. 64, no. 2, pp. 423-431, February 2016, https://doi.org/10.1109/TAP.2015.2505000.

[9] L. Ge, M. J. Li, Y. J. Li, H. Wong, and K. M. Luk, "Linearly polarized and circularly polarized wideband dipole antennas with reconfigurable beam direction," IEEE Transactions on Antennas and Propagation, vol. 66, no. 4, pp. 1747-1755, April 2018, https://doi.org/10.1109/TAP. 2018.2797520 .

[10] F. Wu, K. M. Luk, "Wideband tri-polarization reconfigurable magnetoelectric dipole antenna," IEEE Transactions on Antennas and Propagation, vol. 65, no. 4, pp. 1633-1641, April 2017, https://doi.org/ 10.1109/TAP.2017.2670521.

[11] M.-C. Tang, Z. T. Wu, T. Shi, H. Zeng, W. Lin, and R. W. Ziolkowski, "Dual-linearly-polarized, electrically small, low-profile, broadside radiating, Huygens dipole antenna," IEEE Transactions on Antennas and Propagation, vol. 66, no. 8, pp. 3877-3885, August 2018, https://doi.org/ 10.1109/TAP.2018.2840835.

[12] W. Lin, R. W. Ziolkowski, "Electrically small, low- profile, Huygens circularly polarized antenna," IEEE Transactions on Antennas and Propagation, vol. 66, no. 2, pp. 636-643, February 2018, https://doi.org/ 10.1109/TAP.2017.2784432.

[13] J. O.-Y, Y. M. Pan, and S. Y. Zheng, "Center-fed unilateral and pattern reconfigurable planar antennas with slotted ground plane," IEEE Transactions on Antennas and Propagation, vol. 66, no. 10, pp. 51395149, October 2018, https://doi.org/10.1109/TAP.2018.2860 046.

[14] N. N. Trong, A. Piotrowski, and C. Fumeaux, "A frequencyreconfigurable dual-band low-profile monopolar antenna," IEEE Transactions on Antennas and Propagation, vol. 65, no. 7, pp. 33363343, July 2017, https://doi.org/10.1109/TAP.2017.2702664. 
[15] L. Ge and K. M. Luk, "Frequency-reconfigurable low- profile circular monopolar patch antenna," IEEE Transactions on Antennas and Propagation, vol. 62, no. 7, pp. 3443-3449, July 2014, https://doi.org/10. 1109/TAP.2014.2318077.

[16] A. Mansoul, F. Ghanem, M. R. Hamid, and M. Trabelsi, "A Selective Frequency-Reconfigurable Antenna for Cognitive Radio Applications," IEEE Antennas and Wireless Propagation Letters, vol. 13, pp. 515-518, 2014, https://doi.org/10.1109/LAWP.2014.2311114.

[17] M. W. Young, S. Yong and J. T. Bernhard, "A miniaturized frequency reconfigurable antenna with single bias, dual varactor tuning," IEEE Transactions on Antennas and Propagation, vol. 63, no. 3, pp. 946-951, March 2015, https://doi.org/10.1109/TAP.2015.238 8776.

[18] N. Nguyen-Trong and C. Fumeaux, "Tuning Range and Efficiency Optimization of a Frequency-Reconfigurable Patch Antenna," IEEE Antennas and Wireless Propagation Letters, vol. 17, no. 1, pp. 150-154, Jan. 2018, https://doi.org/10.1109/LAWP.2017.2778272.

[19] A. Petosa, "An Overview of Tuning Techniques for Frequency-Agile Antennas," IEEE Antennas and Propagation Magazine, vol. 54, no. 5, pp. 271-296, Oct. 2012, https://doi.org/10.1109/MAP.2012.6348178.

[20] I. Govardhani et al., "Design of high directional crossed dipole antenna with metallic sheets for UHF and VHF applications," International Journal of Engineering \& Technology, vol. 7, no. 1.5, pp. 42-50, Dec. 2017, https://doi.org/10.14419/ijet.v7i1.5.9120.

[21] D. V. Doan, K. Nguyen, and Q. V. Thai, "A Novel Fuzzy Logic Based Load Frequency Control for Multi-Area Interconnected Power Systems," Engineering, Technology \& Applied Science Research, vol. 11, no. 4, pp. 7522-7529, Aug. 2021, https://doi.org/10.48084/etasr.4320.

[22] N. A. Zainurin, S. a. B. Anas, and R. S. S. Singh, "A Review of Battery Charging - Discharging Management Controller: A Proposed Conceptual Battery Storage Charging - Discharging Centralized Controller," Engineering, Technology \& Applied Science Research, vol. 11, no. 4, pp. 7515-7521, Aug. 2021, https://doi.org/10.48084/ etasr.4217.

[23] Z. A. Shamsan, "Statistical Analysis of 5G Channel Propagation using MIMO and Massive MIMO Technologies," Engineering, Technology \& Applied Science Research, vol. 11, no. 4, pp. 7417-7423, Aug. 2021, https://doi.org/10.48084/etasr.4264.

[24] G. Imamdi, M. V. Narayan, A. Navya, and A. Roja, "Reflector array antenna design at millimetric ( $\mathrm{mm}$ ) band for on the move applications," vol. 13, no. 1, pp. 352-359, Jan. 2018. 